\title{
Functional analysis of SNVs affecting splicing in congenital aniridia
}

\author{
A. Filatova ${ }^{1 *}$, T. Vasilyeva ${ }^{1}$, M. Skoblov ${ }^{1,2}$, A. Voskresenskaya ${ }^{3}$, A. Marakhonov ${ }^{1,2}$, \\ R. Zinchenko ${ }^{1,4}$ \\ ${ }^{1}$ Research Center for Medical Genetics, Moscow, Russia \\ ${ }^{2}$ Moscow Institute of Physics and Technology (State University), Dolgoprudny, Russia \\ ${ }^{3}$ Cheboksary Branch of S. Fyodorov Eye Microsurgery Federal State Institution, Cheboksary, Russia \\ ${ }^{4}$ Pirogov Russian National Research Medical University, Moscow, Russia \\ *e-mail:maacc@yandex.ru
}

Key words: congenital aniridia, PAX6, minigene assay, intronic SNVs, splicing, exon skipping, exon elongation

Motivation and Aim: A congenital aniridia (OMIM 106200) is a rare autosomal dominant panocular disorder caused by mutations in the PAX6 gene or chromosome 11 p13 rearrangements. Haploinsufficiency due to LoF mutations in PAX6 gene is thought to be the main mechanism of congenital aniridia ethiopathogenesis. Mutations affecting splicing are known to be a cause of Mendelian disorders, but their pathogenic status cannot always be provided bioinformatically. This is a reason why some aniridia associated $P A X 6$ nucleotide variants remain outside the mutations database. Minigene splicing assay in vitro allowed us to analyze functional consequences of six different intronic sequence variants, one missense and one synonymous substitution in PAX6 gene found in patients with congenital aniridia.

Methods and Algorithms: DNA samples for analysis were obtained from patients with congenital aniridia (110 patients from 84 unrelated families). The search for mutations in the PAX6 gene was carried out by Sanger sequencing, MLPA and analysis of heterozygosity loss $(\mathrm{LOH})$ in proband. Human Splicing Finder and IntSplice on-line tools were used to predict the effect of identified SNVs on PAX6 pre-mRNA splicing. To confirm the effect of SNVs on splicing experimentally we used in vitro minigene assay. Results: Molecular analysis of a large cohort of aniridia patients from Russia conducted earlier in RCMG revealed a significant proportion of $P A X 6$ mutations affecting splicing $(17.3 \%)$. We focused on 8 SNVs located out of canonical splicing site dinucleotides: 6 deep-intronic and 2 exonic. These variants were classified as variant of unknown significance (VUS), benign or likely pathogenic according to ACMG recommendations. On-line prediction tools analysis revealed that they could influence PAX6 pre-mRNA splicing. In vitro minigene assay showed that all investigated sequence variants except one affected splicing. The variants resulted in open reading frame shifting, premature termination codon formation followed by aberrant mRNA degradation by nonsensemediated decay. Thus, investigated SNVs produce a null allele and haploinsufficiency of the PAX6 function. So investigated mutations were reclassified as loss of function. Conclusion: Using functional in vitro analysis we confirmed the pathogenicity of 7 PAX6 mutations affecting splicing. Our results emphasized the necessity of such an analysis and advanced search for PAX6 mutations.

Acknowledgements: Supported by the RFBR grant (17-04-00475). 